\title{
Relevancy and Manner
}

The Policy Statement titled Gendered and Inclusive Language in the Preparation of Manuscripts: Policy Statement for the Journal of Human Lactation, released by the Journal of Human Lactation (JHL) (Bamberger, et al. 2021), has proposed potentially major changes for manuscripts submitted for publication. As the topic is of universal public health importance, this shift is worthy of closer inspection.

Precision and clarity of language is of primary importance in all exchange of ideas and Grice's Cooperative Principles of Relevance, and Manner are important factors of all communication (See Lewis, 2014). As scientific writing seeks to avoid ambiguity, it is important to investigate how the proposed changes could impact the quality of articles which are submitted to the JHL.

Of major concern is the lack of distinction between sex and gender in the policy statement. The implication of the policy statement as a whole is that social gender is an almost universal attribute of human life and ought to take primacy over biological sex. While gender has existed and is traditionally called a sex-based stereotype, it is not clear from the policy statement if the definition being used is this one--of socially constructed gender. It also raises the question of how important this social construction is to the act of breastfeeding and lactation, an activity that occurs almost universally in one sex. It could be imagined that there are niche articles where it is highly relevant but raising the relevancy as a concern for the processing of all articles could be onerous for many manuscript authors. The cooperative principle of relevancy may be violated in many future submissions.

The policy statement has also run afoul of the Principle of Manner. It may be that the JHL is assuming that there is universal agreement on 'gender', 'gender identity', and 'gender expression.' The definitions of these terms have not been established in linguistics and as scientific writing aims to use terms with agreed upon definitions, it is worth asking if JHL can provide direction to a glossary of definitions for terms or a framework of language use, as other terms, such as 'queer', 'intersex' and 'asexual', have unclear meanings in regards to gender identity. Otherwise, the publication runs the risk of creating ambiguity, leading to a hazard of equivocation when writers attempt to fulfil this new manuscript policy.

The policy also endangers future submissions with vagueness. The policy statement "encourage[s] writers to seek reputable language sources," yet also quotes the American Psychological Association (2020) that "participants may be the most reliable sources in understanding terms they use to describe themselves". Yet terms in gender ideology are still very fluid; both of these statements could struggle to be reliably true in practice. Furthermore, the policy states that some personal referents may be generally offensive and may not be appropriate for publication. However, there is no guideline in what takes which takes precedence, as the document also states, "All gender expression, gender identity, sex and sexual orientation of participants needs to be respectful". It begs the question if respect for the participants or for the readers takes precedence. 
Given that it is already quite frequent in health documents to conflate biological sex and socially constructed gender, there could be further incidences of ambiguity, equivocation, and conflation where clear definitions do not already exist for words and phrases. It is multiplied by the fact that the majority of most populations are not au fait with terms in gender ideology and queer theory. In short, clear definitions expedite the writing and publication process or both parties will fall short of Grice's Principle of Manner.

It is very important of course for all LGBT populations to be addressed by scientific literature and there has been a paucity it--which is not their fault. However, creating carefully planned frameworks to address what may be under-publication is important if relevant and clear literature is to be produced to share information with minimal ambiguity.

Yolanda Forster

MA (Hons) Applied Linguistics and TESOL

\section{References}

Bamberger, E., et al. 2021. Gendered and Inclusive Language in the Preparation of Manuscripts: Policy Statement for the Journal of Human Lactation. Journal of Human Lactation 37(2):227-9. DOI: 10.1177/0890334421995103

Lewis, K. (2014) Philosophy: Language: Gricean Pragmatics.

https://www.youtube.com/watch?v=we6uSVf4qss 